\title{
Evaluación de la vulnerabilidad de edificaciones ante la génesis de lahares: Caso de estudio en la población de Santiago Xalitzintla, en el flanco NE del volcán Popocatépetl (México)
}

Diana Elizabeth Moriano Tello, Paola Andrea Paredes Ruiz, Gustavo Córdoba Guerrero, Hugo Delgado Granados

Diana Elizabeth Moriano Tello

morianoparedesudenar@gmail.com

Paola Andrea Paredes Ruiz Gustavo Córdoba Guerrero

Grupo de investigación en riesgos, amenazas

y medio ambiente GRAMA, Universidad de

Nariño, Ciudad Universitaria, Torobajo, Pasto, Colombia.

\section{Hugo Delgado Granados}

Instituto de Geofísica, Universidad Nacional Autónoma de México UNAM, Circuito de la Investigación Científica S/N, Ciudad Universitaria, Delegación Coyoacán, Ciudad de México.
BOL. SOC. GEOL. MEX. 2017

VOL. 69 NO. 1

P. $223-241$

\section{RESUMEN}

Se analiza la vulnerabilidad estructural a partir de presiones dinámicas debidas al impacto por lahares en la población de Santiago Xalitzintla (México). El proceso de determinación de la vulnerabilidad se realizó de manera independiente a la amenaza (lahar), razón por la cual, se hizo una clasificación para todas y cada una de las construcciones. La tipificación se desarrolló teniendo en cuenta los materiales empleados en la construcción de las edificaciones, y cuánto éstos aportan a su resistencia, de dos maneras. a) Mediante trabajo de campo, con el fin de identificar las características estructurales y las posibles zonas críticas en el lugar. b Utilizando la herramienta Street View de Google Earth como complemento, ya que por diversos factores limitantes durante el reconocimiento directo, no fue posible la identificación de la totalidad de las estructuras.

La vulnerabilidad se estableció de manera semi-cuantitativa, teniendo como base varios estudios que permitieron elaborar un mapa de vulnerabilidad estructural ante daño total para la población, donde se identificó que el $62 \%$ de las edificaciones poseen una vulnerabilidad baja, el $16 \%$ una vulnerabilidad media y el 12 $\%$ una vulnerabilidad alta. Sin embargo, no se lograron identificar algunas construcciones (aproximadamente el 10\% del total) a las cuales se les asignó el rotulo "sin datos".

Mediante el empleo de curvas de probabilidad de destrucción total se elaboraron potenciales situaciones para Santiago Xalitzintla. En éstas, se evidencia que la probabilidad de destrucción total de las estructuras, según su tipología, cambia de acuerdo a las presiones dinámicas, con lo cual es posible evaluar diferentes escenarios de vulnerabilidad en la localidad.

Palabras clave: Vulnerabilidad estructural, Probabilidad de destrucción total, Presión dinámica, Lahar, Volcán Popocatépetl, Santiago Xalitzintla.

\section{ABSTRACT}

Structural vulnerability from dynamic pressures due to the impact of lahars is analyzed in the town of Santiago Xalitzintla (Mexico). The process of vulnerability assessment was conducted independently from the hazard (lahar). For this end, every building was classified. The typification was developed taking into account the materials used in the construction of buildings, and how they contribute to its strength in two ways. a) Based on field work, by visiting the place in order to identify typologies and possible critical areas. b) By using the Google Earth's Street View tool as a complement, since it was not possible to identify all the structures in the visit due to several limiting factors in the direct recognition.

The vulnerability was carried out in a semi-quantitative way, based on several researches which were used to elaborate a structural vulnerability map for total damage. We identified that $62 \%$ of the edifications have low vulnerability, $16 \%$ have medium vulnerability and the remaining $12 \%$ have high vulnerability. However, there were some buildings that could not be identified (around $10 \%$ of total) and were labeled as "no data".

By using total destruction probability curves, potential situations were made for Santiago Xalitzintla. From these curves, it is clear that the total destruction probability of the structures according to their type, changes depending of their dynamic pressures, whereby it is possible to evaluate different vulnerability scenarios on the locality.

Keywords: Structural vulnerability, Probability of total destruction, Dynamic pressure, Lahar, Popocatépetl volcano, Santiago Xalitzintla. 


\section{Introducción}

Los lahares son uno de los fenómenos más destructivos asociados con volcanes, estos presentan altas velocidades, grandes fuerzas de impacto y recorren grandes distancias (Blong, 1984; Tilling y Beate, 1993; Thouret y Lavigne, 2000; Jakob y Hungr, 2005). Se constituyen principalmente por sedimentos volcanogénicos, rocas, barro y agua (Scott et al., 1997; Jenkins et al., 2015). Se clasifican como primarios cuando son desencadenados por actividad volcánica y secundarios cuando se originan por intensas precipitaciones que arrastran material volcánico (Thouret y Lavigne, 2000). Los lahares tienen la capacidad de destruir cualquier tipo de obra civil, además se les atribuye más de 30 mil pérdidas humanas en el siglo XX (Witham, 2005).

En 1877, en la erupción del Volcán Cotopaxi (Ecuador), se originaron lahares producto del derretimiento que sufrió parte de su glaciar al tomar contacto con depósitos de flujos piroclásticos. Éstos causaron serios daños a edificios que se encontraban hasta $240 \mathrm{~km}$ de distancia del Cotopaxi (Miller et al., 1978). Otro evento de gran magnitud fue el ocurrido en Armero (Colombia) en 1985 donde se registró la muerte de más de 23000 personas y dejó un saldo de 5000 afectados (Herd, 1986; Naranjo et al., 1986; Voight, 1996; Thouret et al., 2007; Delgado-Granados et al., 2015). Este hecho se considera como uno de los desastres naturales más devastadores de la historia (Jakob y Hungr, 2005; Huggel et al., 2007); la población fue enterrada por el lahar que se formó luego de la erupción del Nevado del Ruiz (Major y Newhall, 1989). En México, diversas poblaciones han sido afectadas por lahares originados en los flancos del volcán Popocatépetl. Por ejemplo, Siebe et al. (1996a; 1996b), describen la formación de lahares que durante la época prehispánica afectaron comunidades que habitaron alrededor del volcán. Hace 2150 años, flujos de lodo llegaron a la aldea de Tetimpa, población situada en el flanco Nororiental del Popocatépetl (Puebla - México) (Plunket y Uruñuela, 2000; 2005). Este flujo alcanzó un metro de altura y logró inundar toda la región (Seele, 1973; Plunket y Uruñuela, 2008). Igualmente sucedió en la zona arqueológica de Cholula, Cacaxtla y Xochitécatl (México) donde se encontró evidencia de un lahar proveniente del volcán Popocatépetl, similar al anterior ocurrido hace 1100 años (Macías, 2005).

Sobre la Faja Volcánica Transmexicana (FVTM) y entre los límites territoriales de los estados de Morelos, Puebla y Ciudad de México se encuentra el volcán Popocatépetl (Capra et al., 2004), catalogado como uno de los más peligrosos a nivel mundial por los más de 25 millones de personas que habitan en un radio aproximado de $100 \mathrm{~km}$ del cráter (Espinasa, 2014). Se clasifica como un estratovolcán (Aguilera y Ordóñez, 1895; Delgado-Granados y Brugman, 1994) con una actividad intermitente. Después de 70 años inactivo, el 21 de diciembre de 1994 reinicia su actividad eruptiva, la cual se mantiene hasta la actualidad (Macías, 2005; Espinasa, 2014; Delgado-Granados et al., 2015).

Una de las poblaciones que se encuentran amenazadas por la actividad del Popocatépetl es la de Santiago Xalitzintla, ubicada en el Municipio de San Nicolás de los Ranchos en el Estado de Puebla. Se localiza a $2560 \mathrm{msnm}$ y en las coordenadas geográficas $19^{\circ} 0500 \mathbb{N}, 98^{\circ} 30580$ (Carrasco et al., 1985). Por su cercanía al cráter (12 km de distancia) es una de las poblaciones más propensas a ser afectada por algún evento de origen volcánico. Es de notar que la barranca Huiloac atraviesa la localidad, lo cual facilita que los lahares lleguen a la población. Por ejemplo, en marzo de 1997 un flujo de lodo llegó a Santiago Xalitzintla, afectando una casa e inundando lotes con árboles frutales (Sheridan et al., 2001; Capra et al., 2004; Julio et al., 2005).

Volcanes como el Vesubio y Campi Flegrei en Italia, Popocatépetl en México, Sakurajima en Japón y Nevado del Ruiz en Colombia, son escenarios de estudio por la formación de importantes flujos de gravedad; de este modo, los lahares se convierten en una amenaza latente para poblaciones que se encuentran alrededor de volcanes Valentine, 
1998; Thouret et al., 2007). Las consecuencias de un evento eruptivo afectan la vida de seres vivos y causan daños a edificios, infraestructura y vehículos. Para evaluar el riesgo de cualquier amenaza se debe conjugar la amenaza con la vulnerabilidad de los elementos expuestos. En el caso del riesgo por lahares se requiere conocer la vulnerabilidad tanto humana como de las estructuras civiles. La vulnerabilidad de elementos en riesgo por lahares es difícil de evaluar (Ortiz, 1996). En ocasiones no existen suficientes experiencias en las que se determine directamente los daños sobre edificaciones. En general, hay tres formas de evaluar la vulnerabilidad, realizado un análisis: a) Cualitativo, basado en observaciones y clasificando el daño de acuerdo al criterio y experiencia del investigador. b) Cuantitativo, donde las consecuencias de un evento se expresan en costos o en probabilidad de daño por causa de una amenaza. c) Semi-cuantitativo, el cual se basa en relaciones empíricas usando modelos cuantitativos existentes y no simulaciones numéricas (Lee y Jones, 2004; Rivero y Mayorga, 2013). Por ejemplo, Muñoz-Salinas (2007) realizó un análisis semi-cuantitativo de vulnerabilidad de las estructuras presentes en el cauce de la barranca que atraviesa Santiago Xalitzintla, desarrollando un análisis en la región que se consideró como posible zona de inundación.

En este estudio, se analiza la vulnerabilidad de las edificaciones de manera semi-cuantitativa, con base en las presiones dinámicas impartidas por flujos de gravedad y con la clasificación estructural obtenida de diferentes investigaciones. Simultáneamente, se elaboran curvas de probabilidad de daño total, útiles para evaluar diferentes escenarios de respuesta de las edificaciones.

Es importante mencionar que en esta investigación no se considera el efecto de la socavación sobre el suelo de Santiago Xalitzintla debido a que las calles de la población cuentan con revestimiento asfáltico. Además no se contempla el enterramiento dado que solo se evalúa el impacto del lahar sobre las estructuras.

\section{Volcán Popocatépetl}

\subsection{ENTORNO VOLCÁNICO}

El volcán Popocatépetl es un estratovolcán (Aguilera y Ordóñez, 1895; Robin, 1984; Carrasco, 1985) ubicado en la región central de la FVTM, a aproximadamente $65 \mathrm{~km}$ de la Ciudad de México y a unos $45 \mathrm{~km}$ de la ciudad de Puebla. El extremo sur de la Sierra Nevada, se conforma por los volcanes Tláloc, Telapón, Téyotl, Iztaccíhuatl y Popocatépetl (Macías, 2005). Ciclos eruptivos anteriores al edificio del Popocatépetl, dieron lugar a la formación y destrucción de otros volcanes conocidos como el Nexpayantla, el Ventorrillo y el Fraile (Macías, 2005; Espinasa, 2014).

El cono del Popocatépetl está compuesto por la alternancia de depósitos piroclásticos y coladas de lava de composición andesítica-dacítica. La historia geológica del Popocatépetl se sintetiza por ejemplo, en los trabajos expuestos por Macías (2005) y Siebe y Macías (2006).

Desde su reactivación en diciembre de 1994 después de 70 años de inactividad, el volcán Popocatépetl ha tenido etapas explosivas caracterizadas por la formación de domos de lava al interior del cráter (Delgado-Granados et al., 2001). Las cenizas producto de erupciones han alcanzado poblaciones como México y Puebla (Muñoz-Salinas, 2007). En dos ocasiones varios lahares han llegado a la Población de Santiago Xalitzintla producto de la actividad de este volcán (Sheridan et al., 2001; Espinasa, 2014).

El volcán Popocatépetl es monitoreado las 24 horas del día por el Centro Nacional de Prevención de Desastres (CENAPRED) mediante una red de 15 estaciones localizadas en diferentes altitudes hasta de 4300 msnm (Guevara et al., 2003).

\subsection{EVENTOS LAHÁRICOS EN EL FLANGO NE DEL VOLGÁN POPOGATÉPETL}

El volcán Popocatépetl presenta en su flanco NE un gran dinamismo debido a la formación de lahares por la presencia de un glaciar (Palacios, 1995; Delgado-Granados, 1997; Muñoz-Salinas, 
2007; Muñoz-Salinas et al., 2010; Delgado-Granados et al., 2015); además de la creciente actividad eruptiva y la existencia de barrancas (Tenenepanco, La Espinera y Tepeteloncocone) las cuales confluyen sus depósitos en la garganta Huiloac (Muñoz-Salinas, 2007; Muñoz-Salinas et al., 2010) que pasa por las localidades de Santiago Xalitzintla, San Nicolás de los Ranchos, San Buenaventura, Nealtican y San Jerónimo Tecuanipan (Julio et al., 2005).

Es importante considerar el retroceso que ha sufrido el glaciar del volcán Popocatépetl (Palacios, 1996; Delgado-Granados, 1997; Huggel y Delgado-Granados, 2000; Tanarro et al., 2005; Delgado-Granados, 2007; Delgado-Granados et al., 2007; Muñoz-Salinas, 2007). La actividad volcánica y el cambio climático son factores que inciden en el tamaño del glaciar (Delgado-Granados y Brugman, 1994; Delgado-Granados, 1997). Se estima que entre los años de 1989 y 1994 el nivel del glaciar retrocedió aproximadamente $27.5 \mathrm{~m}$ de altura (Palacios, 1996; Palacios et al., 1998). Para el periodo eruptivo iniciado en 1994, hay otro retroceso del glaciar por cuenta de la actividad volcánica con la caída de materiales incandescentes que causaron fusión del casquete (Muñoz-Salinas, 2007). De acuerdo a Tanarro et al. (2005), en el periodo comprendido entre 1982 y 2003 hay una pérdida de $78.4 \%$ de la superficie del glaciar. De este retroceso, se puede inferir que el volumen de los lahares tiende a reducirse.

Desde la reactivación del volcán se presentaron eventos laháricos importantes en los años 1995, 1997 y 2001. De estos eventos, el más considerable se registró en 1997, año en el cual se presentaron lahares producto de la fusión del glaciar y de fuertes precipitaciones (Sheridan et al., 2001), éstos alcanzaron a la localidad de Santiago Xalitzintla y se detuvieron antes de la población de San Nicolás de los Ranchos (Andrés et al., 2014). En el año 2001, se originó un lahar producto de flujos piroclásticos que recorrieron la ladera norte del volcán y lograron desestabilizar el glaciar. Tras un recorrido de $14.3 \mathrm{~km}$ se detuvo a $2 \mathrm{~km}$ de la población de Santiago Xalitzintla (Julio et al., 2008; Andrés et al., 2014; Espinasa, 2014).

Julio y Delgado-Granados (2003) propusieron un método rápido para estimar los flujos laháricos por fusión glacial. Se cuenta además con investigaciones como la de Muñoz-Salinas (2007), Huggel et al. (2007) y Rodríguez et al. (2015) que abordan el tema de lahares desde un enfoque matemático empleando software numérico como LaharZ, MSF y Titan2F. Rodríguez et al. (2015) realiza una aproximación probabilística de la amenaza con el software Titan2F, que combinada con este estudio permitiría una estimación del riesgo por lahares ante destrucción total de las estructuras en la población.

\section{Presión dinámica}

Los lahares son flujos altamente destructivos, esto se debe en parte a la densidad del flujo y su velocidad (Smith y Fritz, 1989; Thouret y Lavigne, 2000; Jenkins et al., 2015). Cuando un flujo impacta sobre una superficie, la fuerza ejercida presenta tres componentes principales conocidas como hidrostática, litostática e hidrodinámica (Jakob et al., 2012), donde las dos primeras dependen de la profundidad del flujo y el espesor de los sedimentos depositados, respectivamente (Zanchetta et al., 2004; Jenkins et al., 2015). En general, la presión dinámica es mayor y se define como la fuerza provocada por una masa y la velocidad con que ésta impacta una superficie (Ecuación 1) (Munson et al., 2006). La aproximación matemática de la presión dinámica se expresa como:

$$
P_{d}=\frac{1}{2} \rho V^{2}
$$

Dónde: $P d$ es la presión dinámica, $\rho$ es la densidad del flujo y $V$ es la velocidad. La densidad de flujo $(\rho)$ (Ecuación 2) depende de la concentración de sedimentos (Streeter et al., 2000; Jenkins et al., 2015). La aproximación matemática de la densidad se expresa como:

$$
\rho=\rho_{s} * \varphi_{s}+\rho_{w} *\left(1-\varphi_{s}\right)
$$


Dónde: $\rho_{w}$ es la densidad del agua, $\rho_{s}$ es la densidad de los sólidos y $\varphi_{s}$ es la fracción volumétrica de sólidos.

El comportamiento de la presión dinámica sobre una estructura puede sintetizarse como "una presión horizontal uniformemente distribuida" sobre el área a la cual impacta (Zúccaro et al., 2012). Los objetos acarreados por el flujo (i.e. mayoritariamente sedimentos de distintos tamaño y ocasionalmente árboles o incluso elementos antrópicos, como pueden ser puentes o vehículos) condicionan significativamente en el grado de impacto sobre un determinado elemento que se localice en su camino, como puede ser una construcción (Jenkins et al., 2015). En estos casos, existe una elevada probabilidad de colapso de una edificación, si la velocidad del flujo es alta (Spence et al., 2004a, 2004b).

\section{Estimación de la vulnerabilidad estructural}

La vulnerabilidad se define como el grado de pérdida (i.e. el daño) de uno o varios elementos, como resultado de la probable ocurrencia de un suceso desastroso o como la predisposición intrínseca de un elemento expuesto a ser afectado o de ser susceptible a sufrir una pérdida (Sandi, 1983; UNDRO, 1991; Barbat y Pujades, 2004)

La vulnerabilidad, frente a las diferentes amenazas volcánicas es difícil de determinar pues se cuenta con pocos datos cuantificados a partir de experiencia directa en erupciones que hayan afectado un área urbana desarrollada (Ortiz, 1996; Araña, 2001; Lee y Jones, 2004). En la actualidad no se cuenta con suficientes estudios que permitan estimar el comportamiento exacto de las estructuras sometidas a fuerzas ejercidas por flujos de gravedad (lahares, flujos piroclásticos, deslizamientos, entre otros).

Estudios sobre el impacto instantáneo como la carga de explosiones nucleares en edificios, pudieran ser usados para el cálculo del daño en obras civiles ocasionados por flujos de gravedad, pues se generan sobrepresiones similares al impacto producido por rocas, troncos, escombros e incluso automóviles, que ponen en peligro la integridad estructural de los edificios (Glasstone y Dolan 1977, Jenkins et al., 2015).

Spence et al. (2004a) logran estimar rangos de daño en las construcciones (valores de resistencia para edificios y elementos de construcción) sometidas a presiones de flujos de gravedad en el área del volcán Vesubio. Ese estudio propone que las estructuras reforzadas son afectadas con presiones dinámicas de 10 a $25 \mathrm{kPa}$. Los autores hacen una clasificación tipológica que abarca tres tipos de construcción encontradas en el lugar. En esta clasificación la vulnerabilidad de las edificaciones depende de la altura, el año de construcción, la cimentación, los materiales empleados y las condiciones actuales de la edificación.

Zanchetta et al. (2004) estudia lo ocurrido en mayo de 1998 en el sur de Italia, donde flujos de escombros generados por deslizamientos debido a intensas lluvias inundaron cerca de $1.38 \mathrm{~km}^{2}$. Se abordan las propiedades físicas y dinámicas de flujos de escombros, donde la componente principal es la velocidad. Los autores concluyen que la forma de una estructura, su orientación respecto al impacto, el número de aberturas (puertas y ventanas) y el tipo de construcción (mampostería o concreto reforzado) son características que inciden significativamente en el comportamiento de la estructura ante el impacto producido por flujos de gravedad. Sin embargo, la existencia de factores como la calidad de la construcción modifica el comportamiento incluso en estructuras con iguales características. Zanchetta et al. (2004) especifica que para presiones dinámicas menores de $35 \mathrm{kPa}$, el daño en las construcciones es de leve a moderado. Por el contrario, el daño es grave cuando la sobrepresión se encuentra entre 35 a $90 \mathrm{kPa}$. Finalmente, concluye que para presiones dinámicas mayores a $90 \mathrm{kPa}$ todos los tipos de estructuras sufren devastación completa.

La investigación realizada por Valentine (1998), estudia el nivel de daño que tienen diferentes estructuras ante el impacto de presión dinámica 
impartida por flujos de gravedad. Este estudio recopila información sobre pruebas nucleares (observaciones y posteriores deducciones) de estudios previos (Pickering y Bockholt, 1971; Glasstone y Dolan, 1977). De acuerdo con Valentine (1998), estos resultados sirven de analogía para flujos de gravedad de origen volcánico. En las tipologías estructurales presentadas por él, se describen los elementos individuales que componen una estructura (ventanas, puertas, muros, techos y pisos) y las edificaciones en su totalidad. En estas tipologías se proponen rangos de probabilidad de destrucción total $(10 \% 50 \%$ y $90 \%)$ para diferentes valores de presión dinámica. Así, para valores de sobrepresión de 7 a $14 \mathrm{kPa}$, el daño producido para construcciones reforzadas y no reforzadas es mínimo o nulo, sin embargo, elementos como ventanas y puertas son seriamente afectados. Si el valor de presión dinámica está entre $20 \mathrm{kPa}$ y 30
$\mathrm{kPa}$, el daño generado a estructuras de concreto reforzado es parcial o incluso severo. Finalmente, considera daño total en cualquier tipo de estructura sometida a presiones mayores de $35 \mathrm{kPa}$. Valentine propone este valor como el límite inferior de destrucción total.

Se ha realizado una compilación de las investigaciones antes analizadas que mediante tipologías estructurales, representan la relación entre presión de impacto y daño. Las características de cada tipología dependen del material empleado en su construcción y de cuánto aportan éstos a su resistencia. En la Figura 1 se aprecia una clasificación de tipologías de 1 a 6 , que corresponde a una composición estructural desde madera hasta acero (Valentine, 1998). Las edificaciones con concreto reforzado en su estructura se clasifican como tipología 3, de la cual se derivan la tipología 3a obtenida del estudio de Spence et al. (2004a), donde

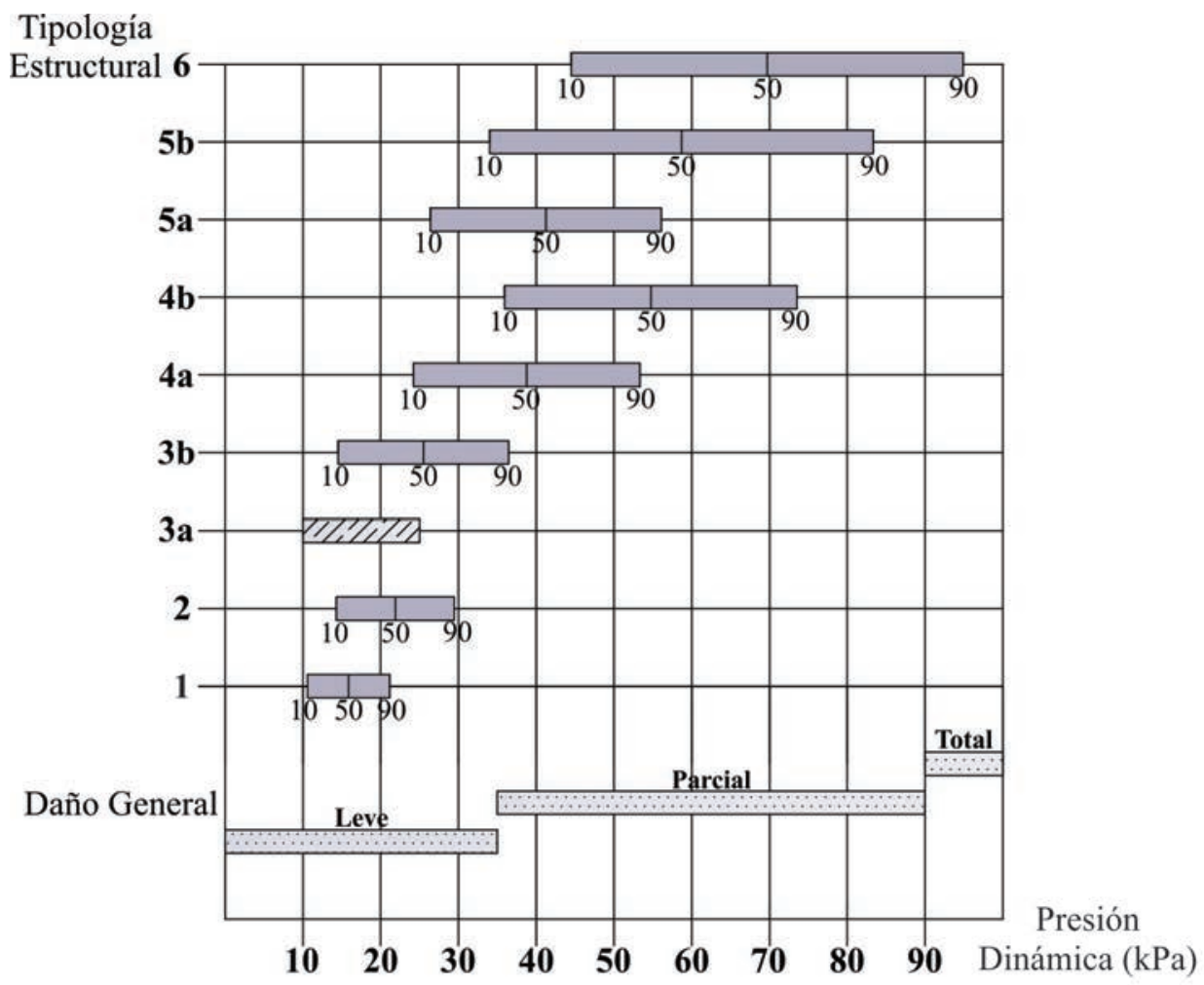

Figura 1 Recopilación de información de las investigaciones realizadas por Valentine (1998); Spence et al. (2004); Zanchetta et al. (2004). Las tipologías 1 - 6 representan lo propuesto por Valentine (color gris). Los atributos que se encuentran en cada tipología (10 \% 50 \% 90 \%) reflejan el porcentaje de probabilidad de destrucción total. La tipología 3a muestra lo expuesto por Spence et al. (2004a) (líneas inclinadas), referente a construcciones reforzadas. En la parte inferior se muestra una escala de daño general en las estructuras propuesta por Zanchetta et al. (2004) (cuadros punteados) clasificado en leve, parcial y total. 
presenta el grado de daño para sobrepresiones de 10 a 25 kPa y la tipología 3b (Valentine, 1998) que expone rangos entre $10 \%$ y $90 \%$ de probabilidad de destrucción total para presiones dinámicas entre 15 y $35 \mathrm{kPa}$. Finalmente, Zanchetta et al. (2004) expone una escala de daño general (leve, parcial y grave) para sobrepresiones entre 0 y 100 $\mathrm{kPa}$. Nótese que Spence et al. (2004a) y Zanchetta et al. (2004) no proponen valores de probabilidad. El análisis de vulnerabilidad que contempla este estudio, se basa en la probabilidad de destrucción total que tienen las estructuras ante sobrepresiones impartidas por flujos de gravedad (lahares), definiendo 3 niveles de vulnerabilidad de acuerdo a las características de las edificaciones y a las sobrepresiones que estas resisten.

\section{Santiago Xalitzintla}

\subsection{DESGRIPGIÓN DEL LUGAR}

Santiago Xalitzintla (Puebla, México) pertenece al municipio de San Nicolás de los Ranchos, con cerca de 2100 habitantes (INEGI, 2000). Esta localidad es una de las más vulnerables por lahares, pues se encuentra en la desembocadura de la barranca Huiloac y es bordeada por la barranca Alseseca (Figura 2b). Se ubica a 12 km del cráter del volcán Popocatépetl (en línea recta) a una altura de 2560 msnm (Muñoz-Salinas, 2007). La población no cuenta con un hospital, ni con una estación de bomberos.

En la Figura 2a se muestra la ubicación del volcán Popocatépetl en México, la localización de Santiago Xalitzintla en el mapa de peligros (Macías et al., 1995), dado que en la actualidad se encuentra en zona de amenaza alta, y finalmente, en la Figura 2b se observan las dos barrancas (Huiloac y Alseseca) que bordean la localidad.

\subsection{INSPECGIÓN DE LA POBLAGIÓN}

En visita de campo realizada en Octubre de 2014, se logró observar la zona donde la barranca Huiloac intercepta por un costado a la población. Las condiciones actuales del terreno muestran que por acción antrópica (i.e. construcción de una cancha) y de la erosión fluvial (Figura 3), el cauce de la barranca ha sido modificado, tal como se aprecia en la Figura $2 b$.

Por medio del trabajo de campo se clasificaron las edificaciones presentes en Santiago Xalitzintla de acuerdo a los estudios expuestos anteriormente. Después de inspeccionar el lugar, se determinó que las construcciones existentes corresponden a las tipologías 1, 2 y 3 b de la Figura 1. Las características de estas tipologías se muestran en la Tabla 1, donde se describe el tipo de edificación y la probabilidad de destrucción total ante diferentes presiones dinámicas. Algunos ejemplos de estas construcciones pueden apreciarse en la Figura 4, donde la Figura 4a (tipología 1) muestra una estructura en estado de deterioro, además los materiales empleados en su construcción ofrecen baja resistencia (muros en madera), la Figura $4 \mathrm{~b}$ (tipología 2) muestra una edificación sin refuerzo, con muros compuestos por bloques y en la Figura 4c (tipología 3b) se observa el estado (adecuado) de una edificación de uso institucional (centro educativo), con refuerzo en su estructura.

Debido a factores que limitaron la accesibilidad, no fue posible la tipificación de la totalidad de las estructuras durante la visita de campo. Para subsanar esta carencia y a fin de complementar la información se recurrió a Google Earth que permite la obtención y sistematización de datos. Mediante la herramienta Street View se visualiza cada edificación presente en la localidad objeto de estudio. Se hace una verificación de la información obtenida en campo (2014) con la información que brinda Google Earth (2009). Los resultados de esta comparación permiten concluir que los cambios encontrados en la localidad con respecto a Google Earth no son significativos, por lo tanto se asume como válido el uso de esta herramienta en este proyecto. En la Figura 5 se examinan 3 casos donde las Figuras 5 a y 5 b muestran dos muros que no presentan ningún cambio. En las Figuras 5c y $5 \mathrm{~d}$ se observa la alcaldía de la localidad con una modificación mínima en su fachada, por último, 

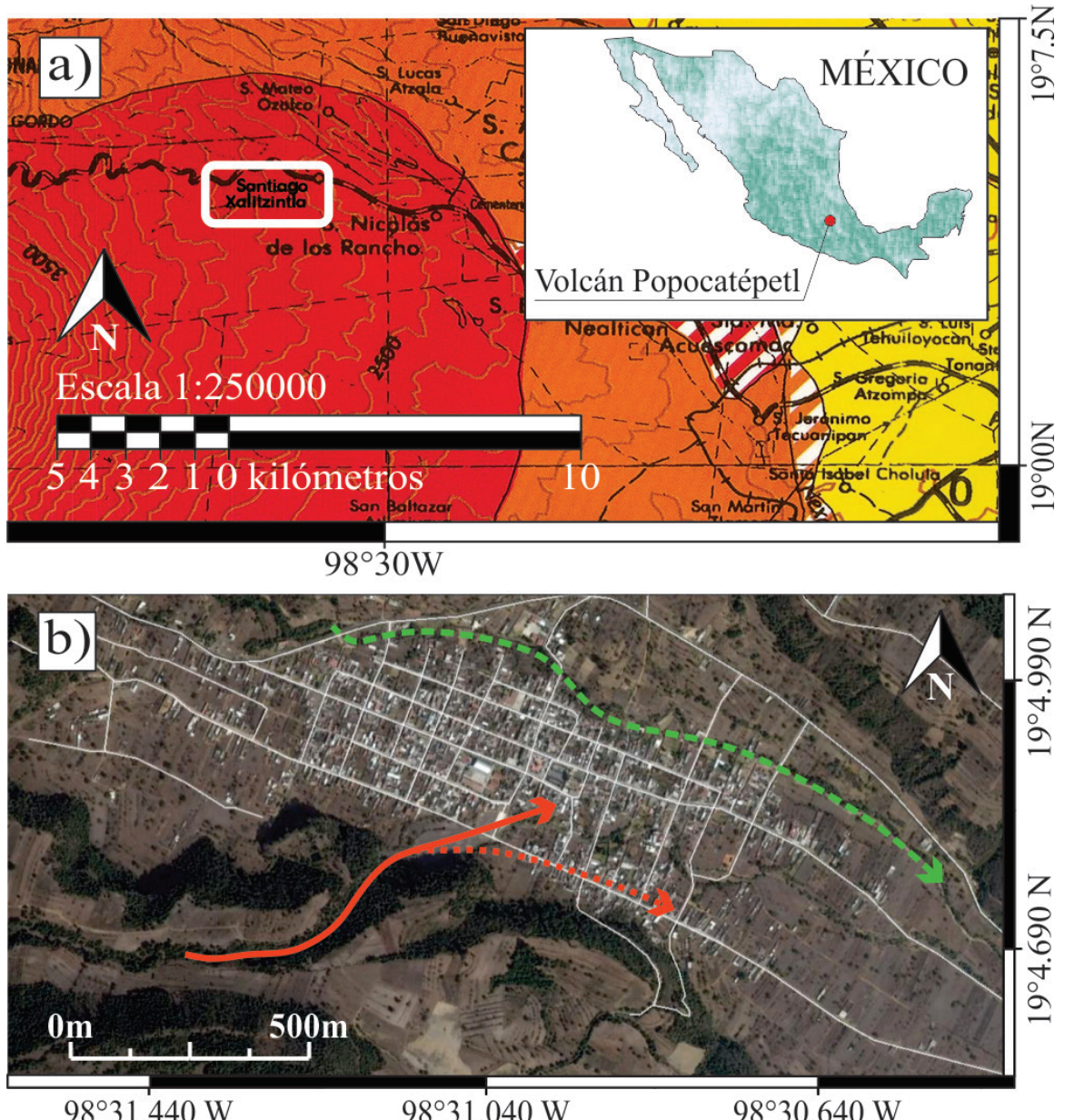

Figura 2 a) El cuadro con el mapa de México muestra la localización del volcán Popocatépetl en el país. En el recuadro blanco se ubica Santiago Xalitzintla en el mapa de peligros del volcán Popocatépetl, modificado de Macías et al. (1995). b) Identificación de las barrancas que interceptan la población (imagen obtenida de Google Earth). La barranca Huiloac se denota con color naranja, donde la línea punteada muestra el cauce anterior mientras que la línea continua indica el cauce actual. El curso de la barranca Alseseca se representa con color verde (línea a trazos).
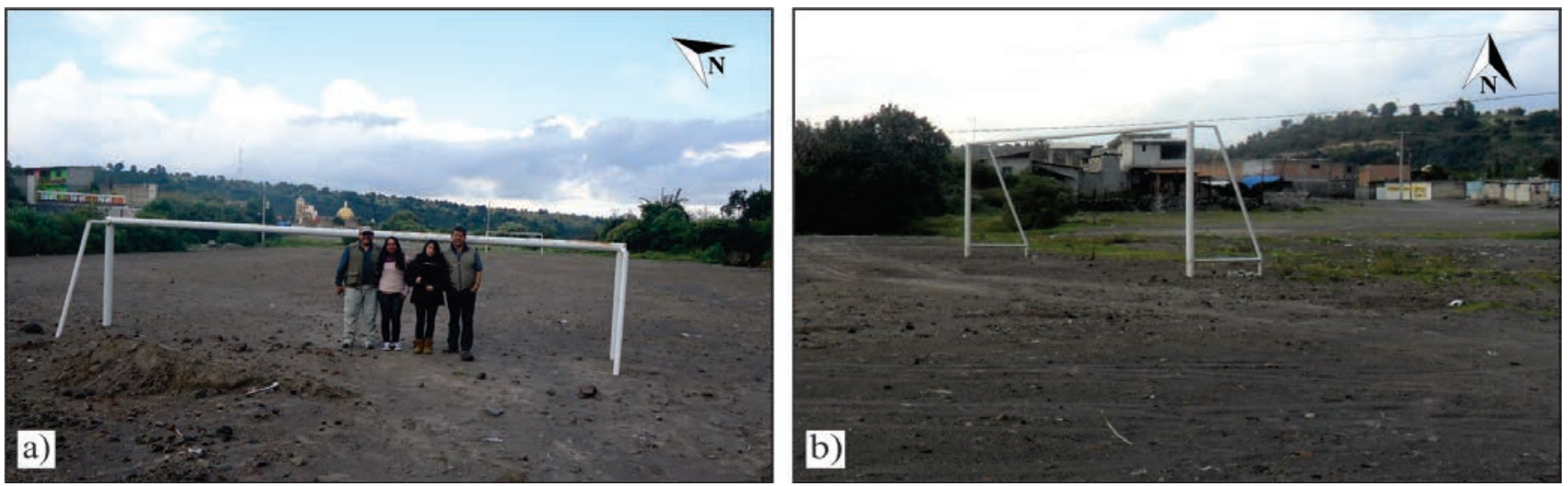

Figura 3 Efectos de la modificación del cauce de la barranca Huiloac. a) Se aprecia el enterramiento parcial del marco de la portería de la cancha oeste, como referencia véase la altura del travesaño con respecto a la altura de las personas. b) Efecto de la erosión fluvial en el marco de portería. La base del arco de la cancha no coincide con el nivel del suelo producto del arrastre de material. 
Tabla 1. Características de las tipologías estructurales (1, 2 y 3b) presentes en Santiago Xalitzintla tomadas de Valentine (1998). Se detalla la composición de las estructuras y los valores de sobrepresión con las que se presenta un 10, 30, 50, 70 y $90 \%$ de probabilidad de destrucción total en las edificaciones. Así para la tipología 2, existe una probabilidad del $30 \%$ de daño total para una presión aproximadamente de $17 \mathrm{kPa}$.

\begin{tabular}{|c|c|c|c|c|c|c|}
\hline TIPOLOGÍA & DESCRIPCIÓN & \multicolumn{5}{|c|}{ PRESIÓN DINÁMICA (kPa) } \\
\hline 1 & $\begin{array}{l}\text { Marco: Viviendas individuales, Casas adosadas, Apartamentos y } \\
\text { hoteles con o sin sótano. Muros: Madera, composición de estuco, } \\
\text { revestimiento de metal, ladrillo y aplacado en piedra y Techo: Plano } \\
\text { o inclinado compuesto por tejas. }\end{array}$ & 10.57 & 13.14 & 15.57 & 18 & 21.17 \\
\hline 2 & $\begin{array}{l}\text { Mampostería No Reforzada: Viviendas individuales, Casas } \\
\text { adosadas, Apartamentos y hoteles con o sin sótano. Muros: } \\
\text { Ladrillo, ladrillo con respaldo de bloques de hormigón o piedra. } \\
\text { Piso: Vigas de madera o de acero, piso en concreto liviano. Techo: } \\
\text { Madera o tejas. }\end{array}$ & 14.3 & 16.98 & 19.86 & 24.8 & 29.47 \\
\hline $3 b$ & $\begin{array}{l}\text { Mampostería Reforzada: Edificios comerciales, residenciales y } \\
\text { oficinas. Muros: Ladrillo macizo, bloque de hormigón o bloque de } \\
\text { hormigón con ladrillo y piedra. Techo: Plano o inclinado } \\
\text { suavemente con viguetas de madera o acero ligero. }\end{array}$ & 14.53 & 17.92 & 21.8 & 29.02 & 36.47 \\
\hline \multicolumn{2}{|r|}{ PROBABILIDAD DE DESTRUCCIÓN TOTAL (\%) } & 10 & 30 & 50 & 70 & 90 \\
\hline
\end{tabular}
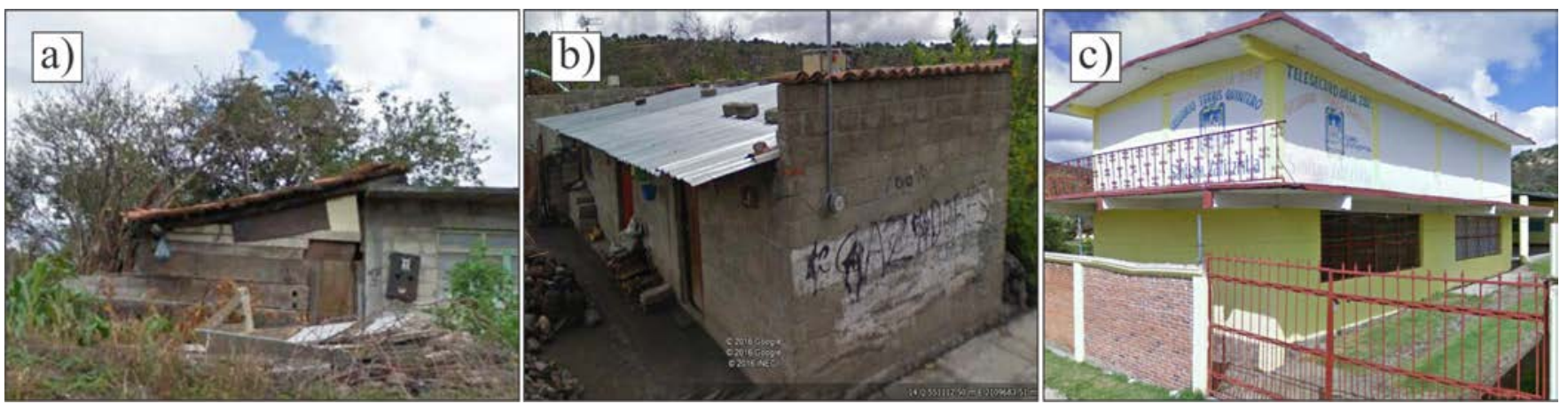

Figura 4 Ejemplos de tipologías estructurales presentes en Santiago Xalitzintla. a) Tipología 1, b) Tipología 2 y c) Tipología 3.

las Figuras 5e y $5 f$ muestran una vivienda en la cual se modificó su estructura con la construcción de una segunda planta.

Confrontando la información de la visita de campo y de Google Earth, con la descripción mostrada en la Tabla 1, se asignaron los atributos correspondientes según el tipo de edificación a cada una de las construcciones localizadas, además se obtuvieron las coordenadas con el fin de usarlas en un sistema de información geográfica para un posterior análisis. En la Figura 6 se muestra la clasificación tipológica de la localidad, donde los colores rojo, naranja y amarillo representan las tipologías 1, 2 y 3 b respectivamente. Las construcciones de uso institucional tales como colegios, alcaldía y puestos de salud, se indican con color azul. Es importante aclarar que algunas zonas no pudieron ser inspeccionadas en la visita de campo y que por algunas limitaciones del programa Google Earth no fue posible tener acceso a determinados lugares, impidiendo su visibilidad. Por lo tanto, a las viviendas que no se logró identificar (aproximadamente 10 $\%$ del total) se les asignó el rotulo de "sin datos". 
Google Earth, 2009
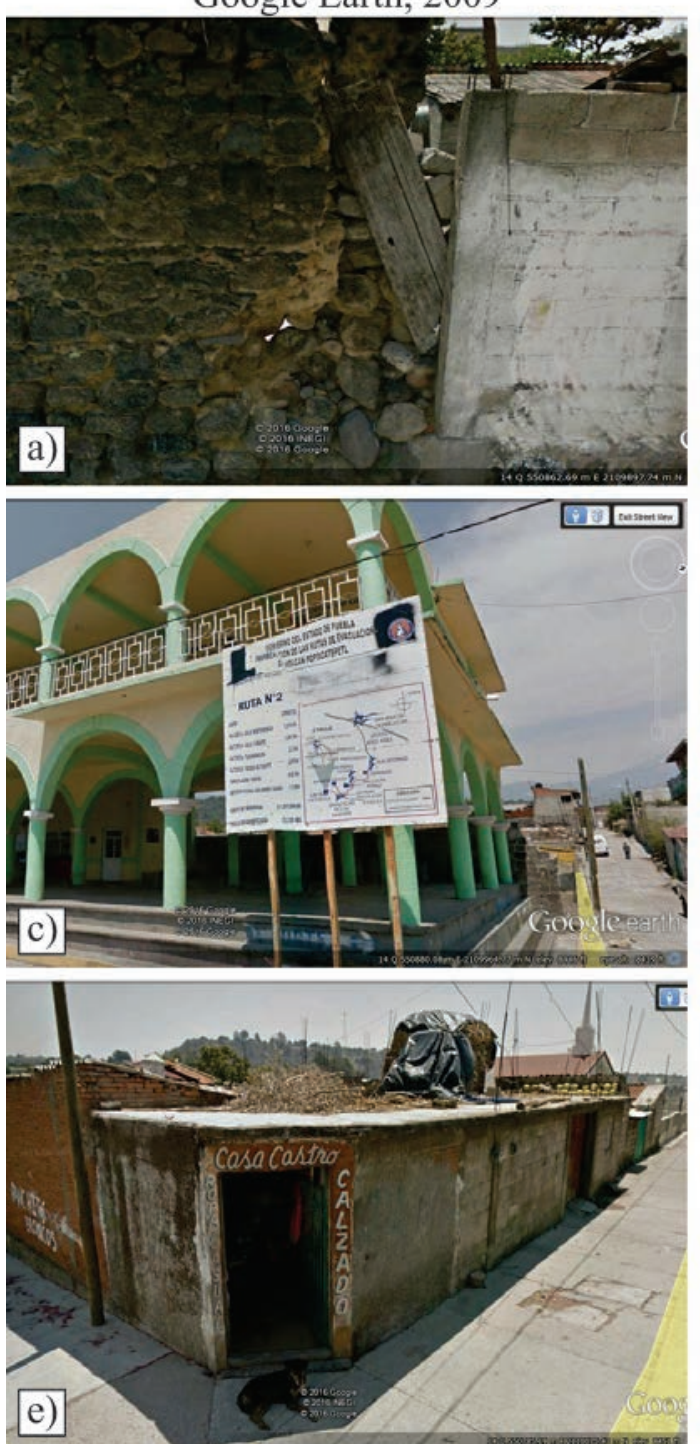

Inspección, 2014
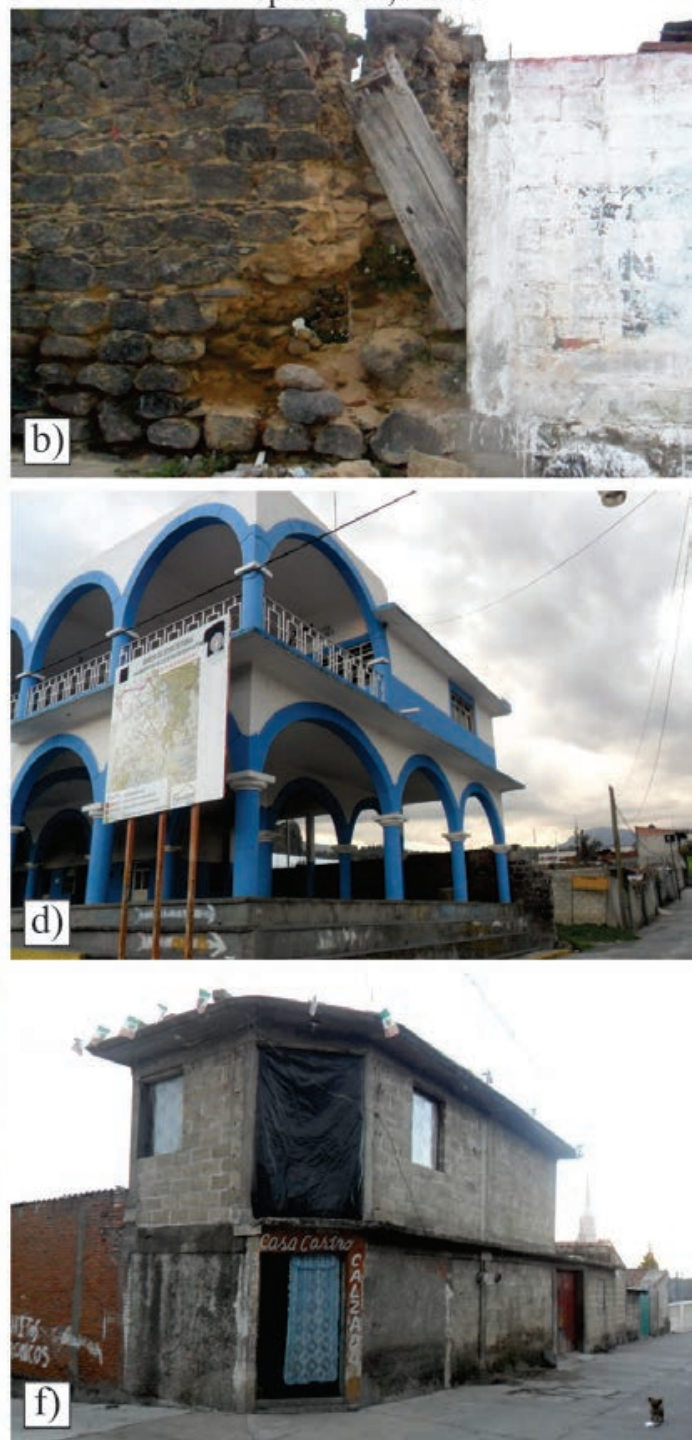

Figura 5 Verificación de diferencias entre Google Earth, 2009 (lado izquierdo) y las mismas edificaciones observadas durante la visita de campo en 2014 (lado derecho). a y b) Estado de dos muros. c y d) Alcaldía. e y f) Modificación estructural en vivienda.

\subsection{MAPA DE LA VULNERABILIDAD ESTRUGTURAL}

De acuerdo a los materiales empleados en la construcción de las edificaciones presentes en Santiago Xalitzintla (Tabla 1) se definen 3 niveles de vulnerabilidad:

1. Baja: Las edificaciones cuyos materiales ofrecen mayor resistencia (mampostería reforzada), y soportan sobrepresiones de hasta 37 $\mathrm{kPa}$.

2. Media: Las edificaciones que no cuentan con refuerzo en su estructura y soportan presiones dinámicas de hasta $30 \mathrm{kPa}$.

3. Alta: Las edificaciones cuyos materiales ofrecen menor resistencia (e.g. madera), y soportan sobrepresiones de hasta $20 \mathrm{kPa}$.

Con la información que proporciona el mapa tipológico (Figura 6) y con los criterios antes expuestos, se define la vulnerabilidad para cada edificación, así, se concluye que las viviendas de tipología 1 tienen una vulnerabilidad alta, las de tipología 2, una vulnerabilidad media y las de tipología $3 \mathrm{~b}$ una vulnerabilidad baja. Para el posterior análisis del riesgo esta información es almacenada en un siste- 
ma de información geográfica (SIG).

El software libre Grass-GIS (Geographical Resources Analysis Support System) es un SIG que permite el procesamiento, análisis y visualización de datos georreferenciados (Neteler et al., 2008). Esta herramienta es utilizada en el análisis del riesgo por lahares del volcán Popocatépetl en la población de Xalitzintla (Rodríguez et al., 2015) y por el programa de modelamiento de lahares Titan2F (Córdoba et al., 2015).

Grass-GIS no es compatible con el formato .kml o .kmz que maneja Google Earth (coordenadas y atributos), por lo tanto fue necesario emplear la aplicación Global Mapper (Global Mapper Software LLC), útil para transformar datos geoespaciales a texto. Con esta extensión, fue posible importar información en la plataforma Grass-GIS para crear una serie de puntos que representan la coordenada y vulnerabilidad de cada edificación, los cuales finalmente fueron exportados al programa Google Earth tras su tratamiento.

Después de este proceso, se obtiene el mapa de vulnerabilidad estructural por daño total de las construcciones de la población de Santiago Xalitzintla, que se puede observar en la Figura 7 donde los colores rojo, naranja y amarillo representan el grado de vulnerabilidad alto, medio y bajo, respectivamente. Se ha denotado con color azul a las zonas comunes (colegios, alcaldía, iglesia, entre otros), ya que al ser centros urbanos concurridos, pueden requerir de un estudio detallado de vulnerabilidad, además, las edificaciones no clasificadas se presentan en color magenta. Del mapa de vulnerabilidad se puede observar que más de la mitad de las edificaciones de la población (62\%) cuentan con refuerzo en su estructura (color amarillo) lo que las hace menos vulnerables.

\section{Evaluación del impacto sobre las estructuras}

Haciendo uso del mapa de vulnerabilidad (Figura 7) y estableciendo valores de presión dentro del rango propuesto en la Figura $1(0 \mathrm{kPa}-100$ $\mathrm{kPa}$ ), es factible determinar posibles escenarios de

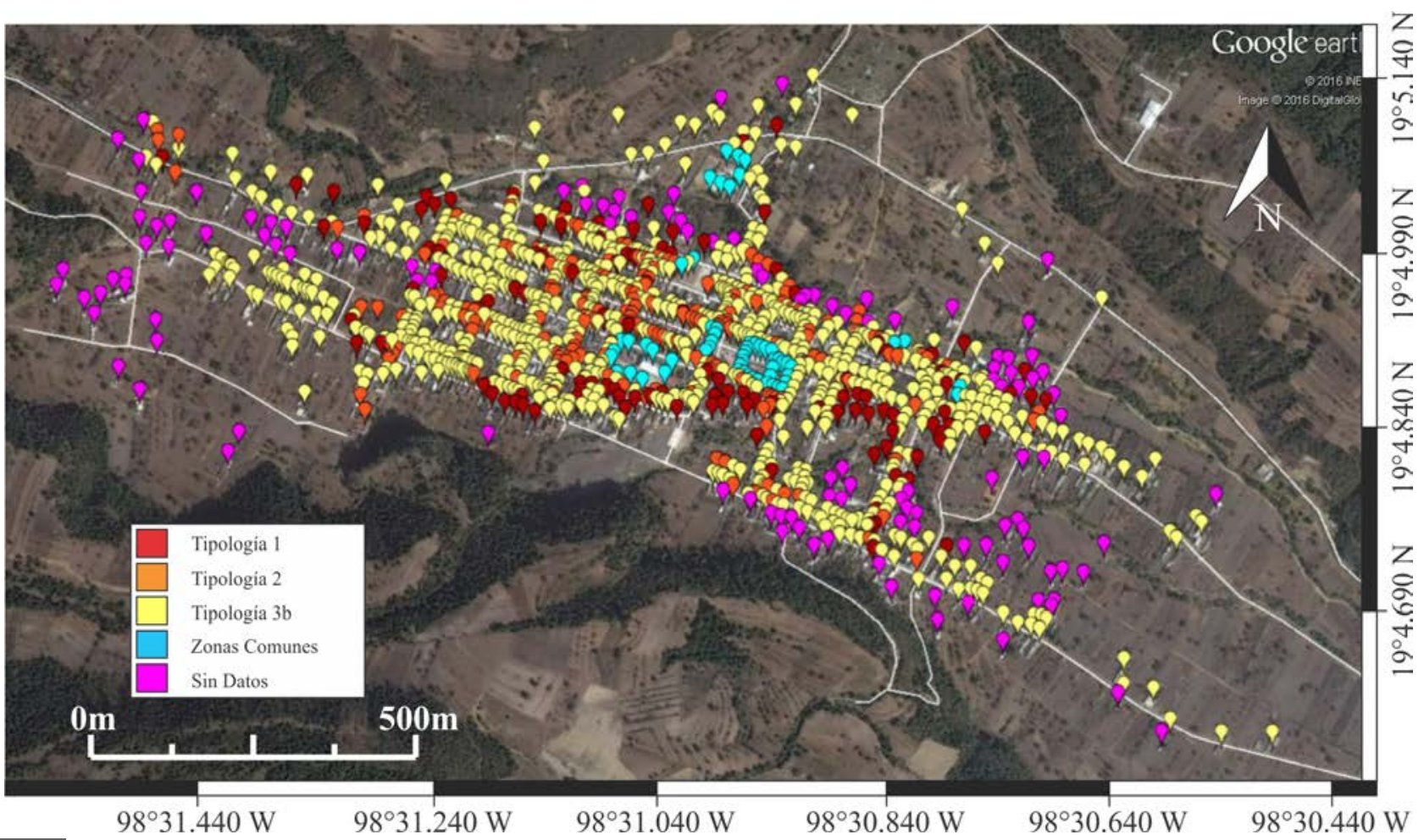




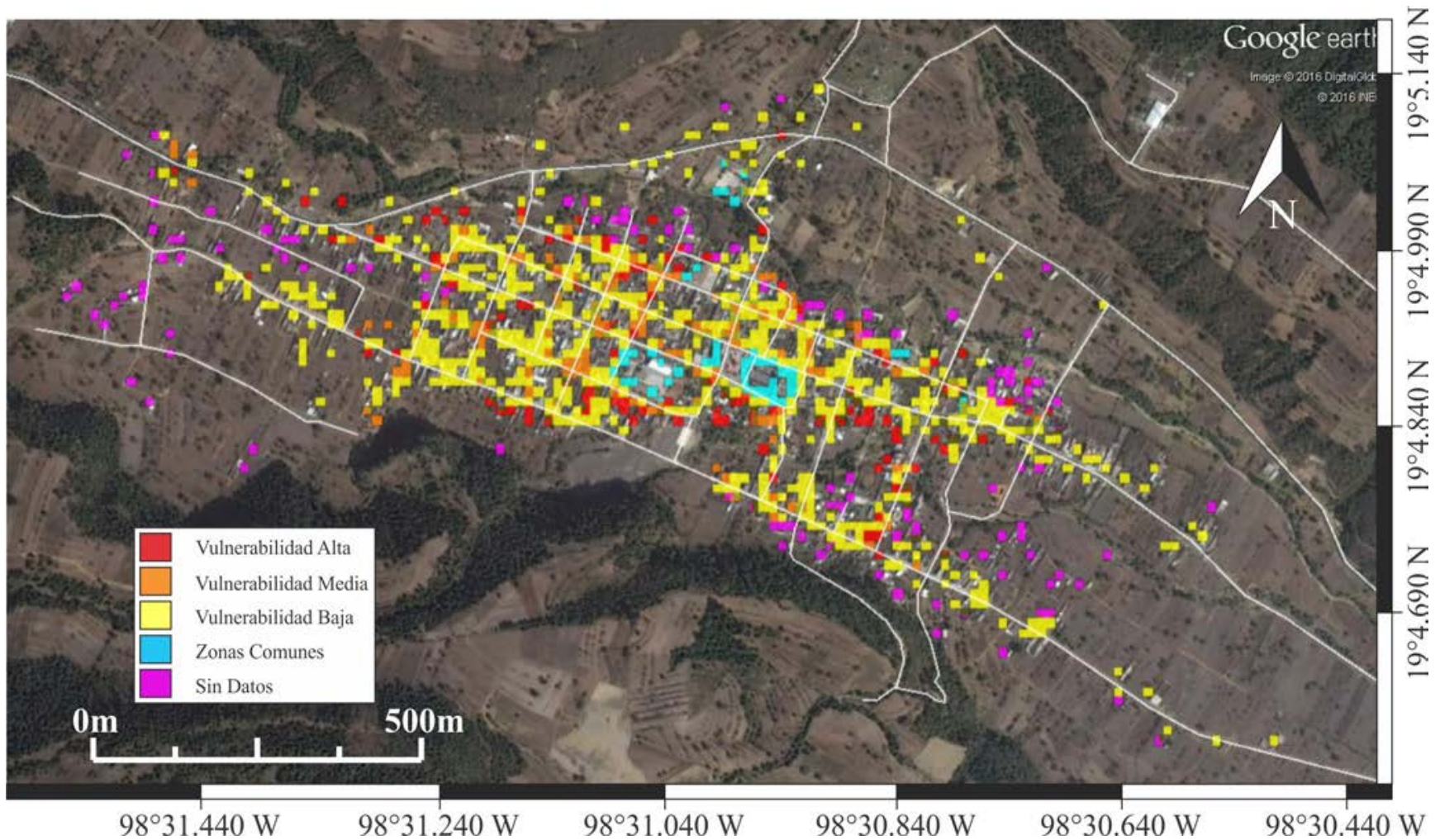

Figura 7 Mapa de vulnerabilidad estructural por daño total de Santiago Xalitzintla. Se define la vulnerabilidad alta, media y baja (color rojo, naranja y amarillo, respectivamente). Las zonas comunes se indican en color azul y las viviendas no identificadas se presentan con color magenta.

respuesta de las estructuras presentes en Santiago Xalitzintla. Con base en la Figura 1 se elaboran curvas de probabilidad de daño total mediante una interpolación cúbica segmentada - spline (Trincado et al., 1999) (Figura 8) que relacionan la probabilidad de destrucción total y presión dinámica para las tipologías estructurales (1, 2 y 3b) propuestas por Valentine (1998). No fue posible realizar este proceso para lo expuesto por Spence et al. (2004a) ni Zanchetta et al. (2004), puesto que en sus trabajos no se proponen valores cuantitativos de probabilidad de destrucción total.

Utilizando las curvas de interpolación de las tipologías 1, 2 y 3b (Figura 8), se elaboran mapas de respuesta estructural para diferentes presiones dinámicas. En este caso se evalúa el comportamiento de las edificaciones en el rango de $10 \mathrm{kPa}$ (presión mínima de afectación para todas las estructuras) a $35 \mathrm{kPa}$ [límite inferior de destrucción para la mayoría de edificaciones según Zanchetta et al. (2004) y Valentine (1998)].

Con el fin de sintetizar la información (Tabla 2) se han empleado rangos de probabilidad del $0-20$ $\%, 20-40 \%, 40-60 \%, 60-80 \%$ y $80-100$ $\%$ representados con los colores azul, verde, amarillo, naranja y rojo respectivamente. Los valores de probabilidad de la Tabla 2, se clasifican en los rangos especificados. De esta forma, el valor de 6 $\%$ se encuentra en el rango $0-20 \%$, por lo tanto se denota con el color azul. Se sigue el mismo proceso para cada uno de los valores de probabilidad. En la Figura 9, se muestran los resultados de Probabilidad de Destrucción Total (PDT) de las estructuras de la localidad frente a cuatro presiones dinámicas evaluadas $(10,20,30$ y $35 \mathrm{kPa})$. En la Figura 9a la PDT es de 0 a $20 \%$ cuando la sobrepresión aplicada es de $10 \mathrm{kPa}$, aquí las estructuras que resultarían afectadas se muestran en color azul. El mapa representado en la Figura 9b muestra el escenario de vulnerabilidad dada una 


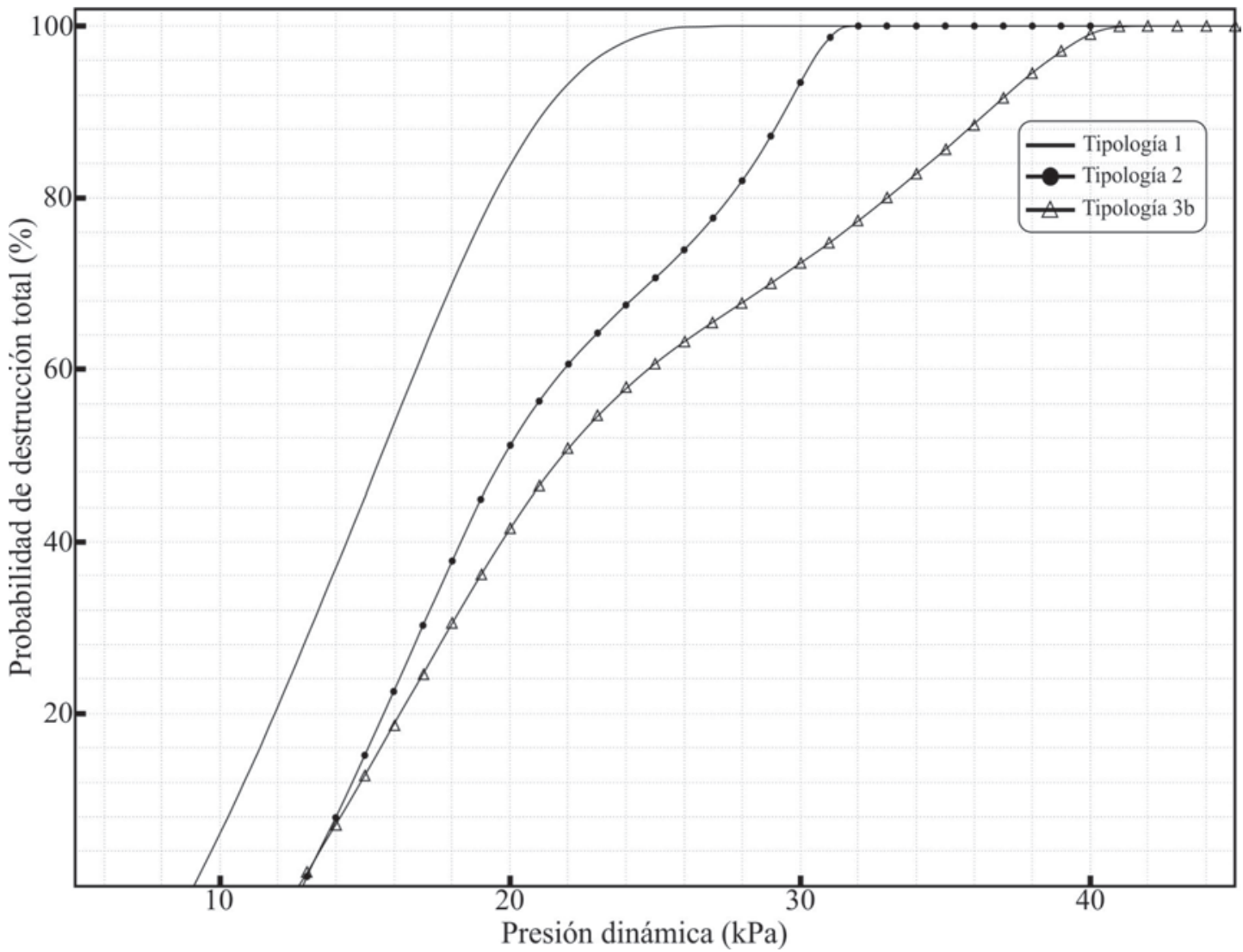

Figura 8 Curva de probabilidad de destrucción total vs presión dinámica, para las tipología 1 (línea continua), 2 (línea con puntos) y 3b (línea con triángulos) deducidas del trabajo de Valentine (1998).

Tabla 2. Se muestran los valores correspondientes de probabilidad de destrucción total para varias sobrepresiones entre 10 y $35 \mathrm{kPa}$. Por ejemplo, para presiones iguales a $20 \mathrm{kPa}$, la tipología 1 presenta un $84 \%$ de probabilidad de destrucción total, la tipología 2 un $51 \%$ y la 3b un $42 \%$.

\begin{tabular}{c|c|c|c|}
\hline $\begin{array}{c}\text { PRESIÓN } \\
\text { DINÁMICA }\end{array}$ & \multicolumn{3}{|c|}{ PROBABILIDAD DE DESTRUCCIÓN TOTAL (\%) } \\
\cline { 2 - 4 }$(\mathbf{k P a})$ & TIPOLOGÍA 1 & TIPOLOGÍA 2 & TIPOLOGÍA 3 \\
\hline 10 & 6 & 0 & 0 \\
\hline 20 & 84 & 51 & 42 \\
\hline 30 & 100 & 93 & 72 \\
\hline 35 & 100 & 100 & 85 \\
\hline
\end{tabular}

presión dinámica de $20 \mathrm{kPa}$, donde la $\mathrm{PDT}$ para las estructuras marcadas en color amarillo se encuentra en un rango de $40 \%$ a $60 \%$. En la Figura 9c correspondiente a $30 \mathrm{kPa}$, la PDT mínima es del $60 \%$, por lo tanto la mayoría de las edificaciones son altamente vulnerables. Finalmente, si la presión dinámica es mayor a $35 \mathrm{kPa}$ las estructuras tendrán un $100 \%$ de PDT (Figura 9d).

\section{Discusión}

En este trabajo se estudia una población que no supera los 3000 habitantes, lo cual ha sido una ventaja en el desarrollo del mismo pues permite hacer una tipificación casa a casa, logrando minimizar las incertidumbres generadas en el proceso de obtención de datos. Sin embargo, para localidades más grandes, es recomendable reducir el tamaño de la muestra identificando primero la región afectada por la amenaza (en este caso, la probabilidad de un lahar) y después proceder a evaluar la vulnerabilidad de las edificaciones en dicha área. Así por ejemplo, en el trabajo de Thouret et al. (2014), se evalúa la vulnerabilidad física de grandes ciudades expuestas a flujos de gravedad, y centra su estudio en Arequipa (Perú). 

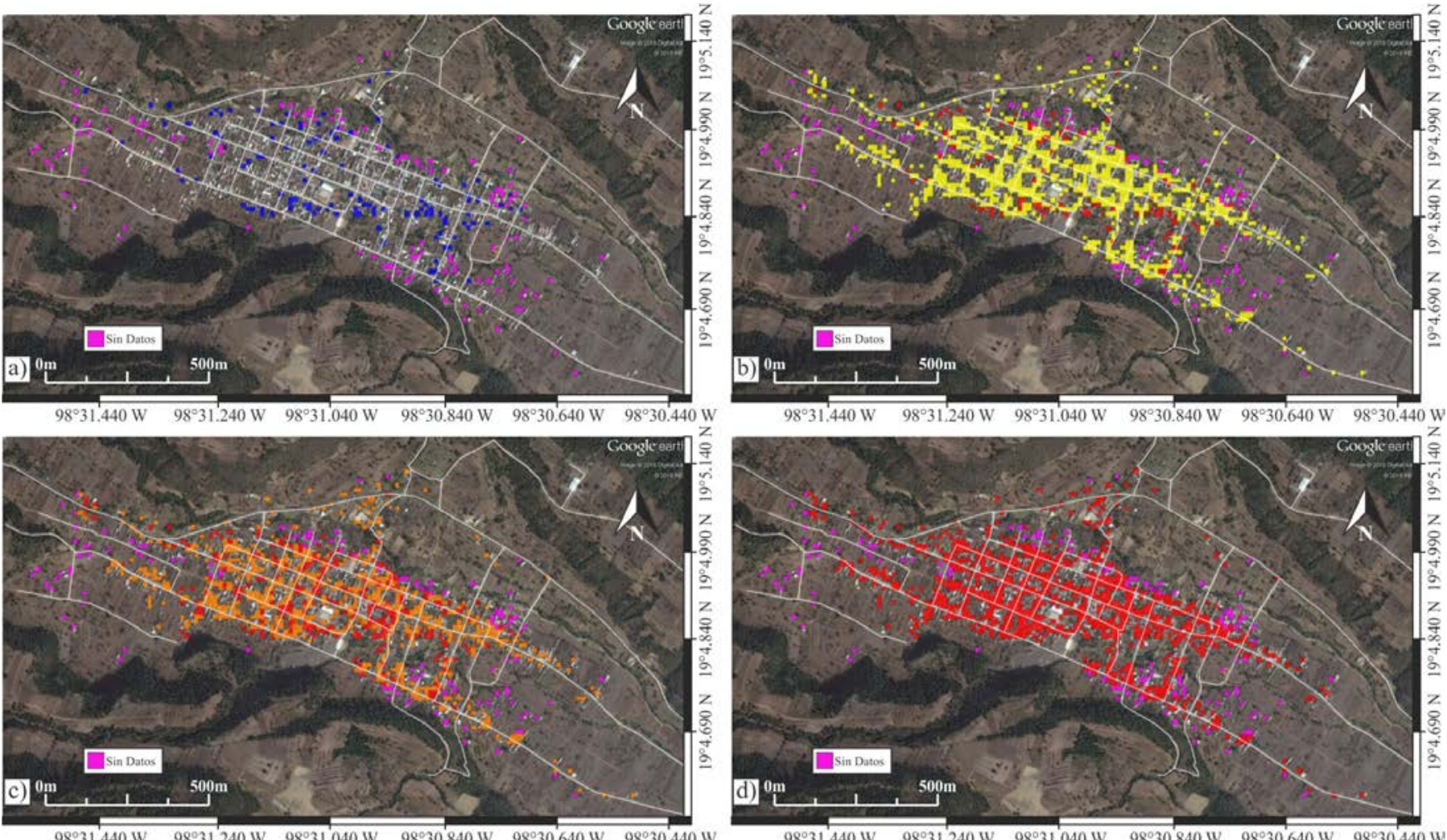

$\begin{array}{llllll}98^{\circ} 31.440 \mathrm{~W} & 98^{\circ} 31.240 \mathrm{~W} & 98^{\circ} 31.040 \mathrm{~W} & 98^{\circ} 30.840 \mathrm{~W} & 98^{\circ} 30.640 \mathrm{~W} & 98^{\circ} 30.440 \mathrm{~W}\end{array}$

$\begin{array}{llllll}98^{\circ} 31.440 \mathrm{~W} & 98^{\circ} 31.240 \mathrm{~W} & 98^{\circ} 31.040 \mathrm{~W} & 98^{\circ} 30.840 \mathrm{~W} & 98^{\circ} 30.640 \mathrm{~W} & 98^{\circ} 30.440 \mathrm{~W}\end{array}$

$0 \%-20 \%$

$20 \%-40 \%$

$40 \%-60 \%$

$60 \%-80 \%$

$80 \%-100 \%$

Figura 9 Mapas de probabilidad de destrucción total para sobrepresiones de a) $10 \mathrm{kPa}$. b) $20 \mathrm{kPa}$. c) $30 \mathrm{kPa}$. d) $35 \mathrm{kPa}$.

Debido al gran tamaño del lugar, los autores estudiaron sólo 1000 edificaciones que se distribuían en 46 cuadras y que se localizaban en zonas que se consideraron potencialmente inundables.

Es importante mencionar que en el trabajo de Valentine (1998) se presentan tipologías estructurales similares a lo propuesto por Spence et al. (2004a), donde se relaciona el daño estructural generado por una presión dinámica para edificaciones reforzadas, siendo diferente el tiempo del impacto que en el caso de Spence, es más prolongado. $\mathrm{Al}$ mismo tiempo Zanchetta et al. (2004), señala que el valor de presión que marca el inicio de afectación severa para la mayoría de las estructuras es de $35 \mathrm{kPa}$, criterio que coincide con lo expuesto por Valentine (1998) quien señala que este valor de presión, es el límite inferior de destrucción total. En el estudio de Jenkins et al. (2015), se sugiere que la carga de explosiones nucleares puede ser usada como analogía para eventos laháricos de magni- tud considerable, ya que se generan presiones dinámicas similares.

Kang y Kim (2016) establecen que para estructuras no reforzadas hay devastación completa a presiones iguales o mayores de $30 \mathrm{kPa}$, lo cual concuerda con lo indicado en la Figura 1 para edificaciones de tipología 2.

$\mathrm{El}$ análisis desarrollado puede ser de ayuda en la gestión y la mitigación del riesgo. No obstante, se sugiere desarrollar modelos de estimación de vulnerabilidad cuantitativa, que dependan de las características propias de edificaciones y lugares donde vayan a ser implementadas, para casos de especial cuidado, como las construcciones catalogadas como zonas comunes o refugios temporales para la población. En todo caso, es recomendable la evacuación ante cualquier evento de origen volcánico. 


\section{Conclusiones}

Se han aplicado varias metodologías para determinar la vulnerabilidad estructural por daño total de las edificaciones presentes en la población de Santiago Xalitzintla, localizada en el estado de Puebla (México). Para esto se analizaron diferentes estudios, de los cuales se recopiló información para determinar que el $62 \%$ de las construcciones ofrecen mayor resistencia al impacto por ser reforzadas (vulnerabilidad baja), sin embargo, cabe resaltar que no se contempló el estado del acero ni su disposición, para determinar estas características se necesita de un estudio mucho más detallado. El $16 \%$ las estructuras de la localidad no cuentan con refuerzo. Por esta razón, su resistencia al impacto es menor y fueron catalogadas como de vulnerabilidad media. Finalmente, el $12 \%$ de las edificaciones son las más vulnerables (tipología 1), debido a que poseen una estructura débil y los materiales empleados en su construcción son de "baja" calidad.

Se determinó como "zona crítica" el lugar donde desemboca la barranca Huiloac en la localidad. Sin embargo, no se delimitó la zona de inundación ya que el análisis de la vulnerabilidad no depende de la amenaza. Así, el proceso de toma de datos se realizó haciendo un análisis visual de cada una de las construcciones de Santiago Xalitzintla obteniendo una coordenada y una tipología en cada edificación.

Por medio de modificaciones de las tablas encontradas en Valentine (1998), se dedujeron curvas de probabilidad de destrucción total que muestran la probabilidad de daño total en las estructuras del lugar ante valores de presión dinámica. El empleo de estas curvas permitió evaluar el comportamiento de las construcciones (mapas de vulnerabilidad) a diferentes presiones dinámicas. Para el caso específico de esta investigación se asignaron presiones de 10 a $35 \mathrm{kPa}$. Finalmente, se concluye que con valores de sobrepresión iguales o mayores a $35 \mathrm{kPa}$ existe daño total en todas las estructuras de Santiago Xalitzintla. Las edificaciones podrían soportar mayores presiones dinámicas si se realiza un reforzamiento en su estructura. Es importante aclarar que se hace un enfoque hacia la vulnerabilidad estructural pero no hacia sus habitantes, razón por la cual se recomendaría reucalizar la población lejos de los cauces de las barrancas.

\section{Agradecimientos}

Los autores agradecemos a la Vicerrectoría de Investigaciones, Postgrados y Relaciones Internacionales (VIPRI) y a la oficina de Bienestar Universitario de la Universidad de Nariño por el financiamiento de este proyecto de investigación. Así mismo, se agradece al Instituto de Geofísica de la Universidad Nacional Autónoma de México (UNAM) por hacer posible el trabajo de campo en Santiago Xalitzintla. Igualmente, agradecemos a la Dra. Esperanza Muñoz Salinas y al jurado anónimo por las observaciones que permitieron mejorar este trabajo y a la revisión previa de ingeniero Roberto Torres del Servicio Geológico Colombiano (SGG) y al ingeniero Michel Bolaños de la Universidad de Nariño.

\section{Referencias}

Aguilera, J., Serrano, J., Ordóñez, E., 1895, Expedición científica al Popocatépetl: México, Oficina de la Secretaría de fomento, Comisión Geológica Mexicana, 48 p.

Andrés, N., Zamorano, J., Blasco, J., Tanarro, M., Palacios, D., 2014, Evolución post-lahárica de un canal pro glaciar, garganta de Huiloac (México): Boletín de la Sociedad Geológica Mexicana, 66 (2), 305-328.

Araña, V., 2001, Volcanismo de las Islas Canarias, en Astiz, M., García, A. (eds.), Curso Internacional de Volcanología y Geofísica Volcánica: Madrid, Cabildo de Lanzarote, 105-114.

Barbat, A., Pujades, L., 2004, Evaluación de la Vulnerabilidad y del riesgo sísmico en zonas urbanas. Aplicación a Barcelona (resumen), 
en Sexto Congreso Nacional de Sismología e Ingeniería Sísmica: Guimarães, Portugal, Sísmica 2004, 229-252.

Blong, R., 1984, Volcanic hazards, a sourcebook on the effects of eruptions: Australia, Academic Press, 424 p.

Capra, L., Poblete, A., Alvarado, R., 2004, The 1997 and 2001 lahars of Popocatépetl volcano (Central México) textural and sedimentological constraints on their origin and hazards: Journal of Volcanology and Geothermal Research, 131(3), 351-369.

Carrasco, G., 1985, Estudio geológico del Volcán Popocatépetl: México D.F., Universidad Nacional Autónoma de México, Facultad de Ingeniería, Tesis profesional, $41 \mathrm{p}$.

Carrasco, G., Silva, L., Delgado, H., Urrutia, J., 1985, Geología y paleomagnetismo del volcán Popocatépetl (resumen), en Memorias de la Reunión Anual de la Unión Geofisica Mexicana: México, Unión Geofísica Mexicana, 205-225.

Córdoba, G., Villarosa, G., Sheridan, M., Viramonte, J., Beigt, D., Salmuni, G., 2015, Secondary lahar hazard assessment for Villa la Angostura, Argentina, using Two-PhaseTitan modelling code during 2011 Cordón Caulle eruption: Natural Hazards and Earth System Science, 15(4), 757-766.

Delgado-Granados, H., Brugman, M., 1994, Monitoreo de los glaciares del Popocatépetl, en Centro Nacional de Prevención de Desastres (ed.), Volcán Popocatépetl, estudios realizados durante la crisis de 1994 - 1995: México, Secretaría de Gobernación, 221-241.

Delgado-Granados, H., 1997, The glaciers of Popocatépetl volcano (Mexico): Changes and causes. Quaternary International, 43, 53-60. Delgado-Granados, H., Piedad, N., Cárdenas, L., 2001, Sulfur dioxide emissions from Popocatépetl volcano (Mexico): Case study of a high-flux passively-degassing erupting volcano: Journal of Volcanology and Geothermal Research, 108, 107-120.
Delgado-Granados, H., 2007, Climate change vs. Volcanic Activity: Forcing Mexican Glaciers to Extinguish and Related Hazards. Proceedings of the First International Conference on the Impact of Climate Change on High-Mountain Systems: Bogotá, Colombia, Instituto de Hidrología, Meteorología y Estudios Ambientales, 153-168.

Delgado-Granados, H., Julio, P., Huggel, C., Ortega, S., Alatorre, M., 2007, Chronicle of a death foretold: Extinction of the smallsize tropical glaciers of Popocatépetl volcano (Mexico): Global and Planetary Change, 56, $13-22$.

Delgado-Granados, H., Julio, G., Ontiveros, J., Cortés, G., Carrasco, B., Pulgarín, P., Mothes, H., Moreno, B., Cáceres E., Correa, B., 2015, Hazards at Ice-clad volcanoes, en Haeberli, W. (ed.), Snow and Ice-Related Hazards, Risks, and Disasters: Amsterdam, Elsevier Book Series on Natural Hazard, 607-636.

Espinasa, R., 2014, Historia de la actividad del volcán Popocatépetl - 17 años de erupciones (en línea): Centro Nacional de Prevención de Desastres, México, disponible en <www. cenapred.gob.mx>, acceso libre, consultado 15 de julio 2015.

Glasstone, S., Dolan, P., 1977, The effects of nuclear weapons: Washington DC, U.S.A., Department of Defense and Department of energy, report, $653 \mathrm{p}$.

Guevara, E., Quaas, R., Castelán, G., Ortiz, J., Vázquez, J., Morquecho, C., Alarcón, A., Martínez, A., Gómez, A., Espitia, G., Alonso, P., Cárdenas, L., 2003, Instrumentación y monitoreo del volcán Popocatépetl: México, Centro Nacional de Prevención de Desastres, Informes Técnicos, $102 \mathrm{p}$.

Herd, G., 1986, The 1985 Ruiz volcano disaster: Eos, Transactions American Geophysical Union, 67(19), 457-460.

Huggel, C., Delgado-Granados, H., 2000, Glacier monitoring at Popocatépetl volcano, 
Mexico: Glacier shrinkage and possible causes, en Hegg, C., Vonder Muehll, D. (eds.), Beitraege zur Geomorphologie, Proceedings der Fachtagung der Schweizerischen Geomorphologischen Gesellschaft vom 8.-10. Juli 1999 in Bramois (Kt. Wallis), Birmensdorf, Eidgenoessische Forschungsanstalt WSL, 97-106.

Huggel, C., Ceballos, L., Pulgarín, B., Ramírez, J., Thouret, J.G., 2007, Review and reassessment of hazards owing to volcanoglacier interactions in Colombia: Annals of Glaciology, 45(1), 128-136.

Instituto Nacional de Estadística, Geografia e Informática (INEGI), 2000, México: XII Censo General de Población y Vivienda 2000, Tabulados de la muestra censal, Cuestionario ampliado.

Jakob, M., Hungr, O., 2005, Debris-flow hazards and related phenomena: Springer, 411-443.

Jakob, M., Stein, D., Ulmi, M., 2012, Vulnerability of buildings to debris flow impact: Natural Hazards, 60(2), 241-261.

Jenkins, F., Phillips, C., Price, R., Feloy, K., Baxter, P., Hadmoko, D., Bélizal, E., 2015, Developing building-damage scales for lahars: application to Merapi volcano, Indonesia: Bulletin of Volcanology, 77(9), $1-17$.

Julio, P., Delgado-Granados, H., 2003, Fast hazard evaluation employing digital photogrammetry: Popocatépetl glaciers, Mexico: Geofísica Internacional, 42(2), 275-283.

Julio, P., González, A., Delgado-Granados, H., Kääb, A., 2005, Glacier melting formation during January 22, 2001, eruption Popocatépetl volcano (Mexico): Zeitschriftfür Geomorphologie, 140, 95-102.

Julio, P., Delgado-Granados, H., Huggel, C., Kääb, A., 2008, Impact of the eruptive activity on glacier evolution at Popocatépetl volcano (México) during 1994-2001: Journal of Volcanology and Geothermal Research, 170, 86-98.
Kang, H., Kim, Y., 2016, The physical vulnerability of different types of building structure to debris flow events: Natural Hazards, 80(3), 1475-1493.

Lee, E., Jones, K., 2004, Landslide risk assessment: London, Thomas Telford Publishing, 275 p.

Macías, J., Carrasco, G., Delgado, H., Martin, A., Siebe, C., Hoblitt, R., Tilling, R., 1995, Mapa de peligros del volcán Popocatépetl, (en línea): México, Instituto de Geofísica Universidad Nacional Autónoma de México (UNAM), disponible en <http://www. geofisica.unam.mx/>, consultado 22 de agosto de 2015.

Macías, J., 2005, Geología e historia eruptiva de algunos de los grandes volcanes activos de México: Boletín de la Sociedad Geológica Mexicana, volumen Conmemorativo del Centenario, Temas Selectos de la Geología Mexicana, 57(3), 379-424.

Major, J., Newhall, C., 1989, Snow and ice perturbation during historical volcanic eruptions and the formation of lahars and floods: Bulletin of volcanology, 52(1), 1-27.

Miller, C., Mullineaux, D., Hall, M., 1978, Mapa de reconocimiento de riesgos volcánicos potenciales del volcán Cotopaxi, 1:100000: United States Geological Survey, miscell invest, Map 1-1072.

Munson, B., Young, D., Okiishi, T., 2006, Fundamentals of Fluid Mechanics (5 edition): U.S.A., John Wiley \& Sons, Inc., 816 p.

Muñoz-Salinas, E., 2007, Los lahares del Popocatépetl: Obtención y tratamiento de la información para la prevención de riesgos: España, Universidad Complutense de Madrid, tesis doctoral, $236 \mathrm{p}$.

Muñoz-Salinas, E., Castillo, M., Manea, V., Palacios, D., 2010, On the geochronological method versus flow simulation software application for lahar risk mapping: A case study or Popocatépetl volcano, Mexico: Geograsfika Annaler, Series A. Physical Geography, 92(3), 311-328. 
Naranjo, J., Sigurdsson, H., Carey, S., Fritz, W., 1986, Eruption of the Nevado del Ruiz volcano, Colombia, on 13 November 1985: tephra fall and lahars: Science, 233(4767), 961-963.

Neteler, M., Beaudette, D., Cavallini, P., Lami, L., Cepicky, J., 2008, GRASS GIS, en Hall, G., Brent y Leahy, Michael, G. (eds.), Open Source Approaches in Spatial Data Handling: Berlin, Springer, 171-199.

Ortiz, R., 1996, Riesgo volcánico: España, Servicio de publicaciones del Excmo, Cabildo Insular de Lanzarote, 329 p.

Palacios, D., 1995, Rockslide processes on the north slope of Popocatépetl Volcano, Mexico: Permafrost and Periglacial Processes, 6(4), John Wiley and Sons Ltd, 281-379.

Palacios, D., 1996, Recent geomorphologic evolution of a glaciovolcanic active stratovolcano Popocatépetl (México): Geomorphology, 16(4), 319-335.

Palacios, D., Zamorano, J., Parrilla, G., 1998, Proglacial debris flows in Popocatépetl north face and their relation to 1995 eruption: Zeitschrift fur Geomorphologie, 42(3), 273-295.

Pickering, E., Bockholt, J., 1971, Probabilistic air blast failure criteria for urban structures: U.S.A., Stanford Research Inst Menlo Park $\mathrm{Ca}$, reporte técnico, $110 \mathrm{p}$.

Plunket, P., Uruñuela, G., 2000, The archaeology of a Plinian eruption of the Popocatépetl volcano: Geological Society, London, Special Publications, 171(1), 195-203.

Plunket, P., Uruñuela, G., 2005, Recent research in Puebla prehistory: Journal of Archaeological Research, 13(2), 89-127.

Plunket, P., Uruñuela, G., 2008, Mountain of sustenance, mountain of destruction: The prehispanic experience with Popocatépetl volcano: Journal of Volcanology and Geothermal Research, 170(1), 111-120.

Rivero, N., Mayorga, A., 2013, Evaluación cuantitativa de la vulnerabilidad física de estructuras de uno y dos pisos por caídas de roca: Bogotá, Pontificia Universidad Javeriana, tesis de pregrado, $143 \mathrm{p}$.

Robin, C., 1984, Le Volcan Popocatepetl (Mexique): structure, evolution pétrologique et risques: Bulletin volcanologique, 47(1), $1-23$.

Rodríguez, D., Moriano, D., Paredes, P., Córdoba, G., Delgado, H., 2015, Evaluación del Riesgo por lahares provenientes del volcán Popocatépetl, en la población de Xalitzintla, Puebla, México (resumen), en Foro Internacional sobre Gestión del Riesgo Geológico, Arequipa, Perú: Arequipa, Jersy Mariño, J., Villacorta, S. (eds.), 295 p.

Sandi, H., 1983, Earthquake risk and earthquake preparedness: some qualitative aspects and quantification possibilities (seminar), in Project for Earthquake Risk Reduction in the Balkan Region, Geneva, Office of the United Nations Disaster Relief Co-coordinator (UNDRO), 79-93.

Scott, W., Pierson, T., Schilling, S., Costa, J., Gardner, C., Vallance, J., Major, J., 1997, Volcano hazards in the Mount Hood region, Oregon: U.S.A., U. S. Department of the Interior, US Geological Survey, Report, 16 p. Seele, E., 1973 , Restos de milpas y poblaciones prehispánicas cerca de San Buenaventura Nealtican, Puebla: Comunicaciones, 7, 77-86.

Sheridan, M., Hubbard, B., Bursik, M., Abrams, M., Siebe, C., Macías, J., Delgado, H., 2001, Gauging short-term volcanic hazards at Popocatépetl: EOS, Transactions American Geophysical Union, 82(16), 185-189.

Siebe, C., Macías, J., Abrams, M., Obenholzner, J., 1996a, La destrucción de Cacaxtla y Cholula, Un suceso en la historia eruptiva del Popocatépetl: Revista Ciencias, Facultad de Ciencias, Universidad Nacional Autónoma de México, v. 41, 36-45.

Siebe, C., Abrams, M., Macías, J., Obenholzner, J., 1996b, Repeated volcanic disasters in Prehispanic time at Popocatépetl, central Mexico Past key to the future?: Geology, 
24(5), 399-402.

Siebe, C., Macías, J., 2006, Volcanic hazards in the Mexico City metropolitan area from eruptions at Popocatépetl, Nevado de Toluca, and Jocotitlán stratovolcanoes and monogenetic scoria cones in the Sierra Chichinautzin Volcanic Field: Geological Society of America Special Papers, 402, 253-329.

Smith, G., Fritz, W., 1989, Volcanic influences on terrestrial sedimentation: Geology, 17(4), 375-376.

Spence, R., Baxter, P., Zuccaro, G., 2004a, Building vulnerability and human casualty estimation for a pyroclastic flow, a model and its application to Vesuvius: Journal of Volcanology and Geothermal Research, 133(1), 321-343.

Spence, R., Zuccaro, G., Petrazzuoli, S., Baxter, P., 2004b, Resistance of buildings to pyroclastic flows, analytical and experimental studies and their application to Vesuvius: Natural Hazards Review, 5(1), 48-59.

Streeter, V., Wylie, E., Bedford, K., 2000, Mecánica de los fluidos (Vol. 9): Santafé de Bogotá, McGraw-Hill, 740 p.

Tanarro, L., Zamorano, J., Palacios, D., 2005, Glacier degradation and lahar formation on the Popocatepetl volcano (Mexico) during the last eruptive period (1994-2003): Zeitschriftfür Geomorphologie, 140, 73-92.

Thouret, J., Lavigne, F., 2000, Lahars: occurrence, deposits and behaviour of volcanohydrologic flows, en Leyrit, H., Montenat, C. (eds.), Volcaniclastic rocks from magma to sediments: Amsterdam, Gordon and Breach Science Publishers, 151-174.

Thouret, J., Ramírez, J., Gibert, B., Vargas, C., Naranjo, J., Vandemeulebrouck, J., Valla, F., Funk, M., 2007, Volcano-glacier interactions on composite cones and lahar generation, Nevado del Ruiz, Colombia, case study: Annals of Glaciology, 45(1), 115-127.
Thouret, J., Ettinger, S., Guitton, M., Santoni, O., Magill, C., Martelli, K, Zuccaro G, Revilla V, Charca J. Arguedas, A., 2014, Assessing physical vulnerability in large cities exposed to flash floods and debris flows, the case of Arequipa (Peru): Natural Hazards, 73(3), 1771-1815.

Tilling, R., Beate, B., 1993, Los peligros volcánicos. Apuntes sobre un curso breve: Organización Mundial de Observatorios Vulcanológicos, $1-13$.

Trincado, G., Vidal, J., 1999, Aplicación de interpolación "spline" cúbica en la estimación de volumen, Chile: Bosque, 20(2), 3-8.

United Nations Disaster Relief Organization (UNDRO), 1991, Mitigating Natural Disasters: Phenomena, Effects and Options: New York, U.S.A., United Nations, A Manual for Policy Makers and Planners, 164 p.

Valentine, G., 1998, Damage to structures by pyroclastic flows and surges, inferred from nuclear weapons effects: Journal of Volcanology and Geothermal Research, 87(1), 117-140.

Voight, B., 1996, The management of volcano emergencies: Nevado del Ruiz, in Monitoring and mitigation of volcano hazards: Berlin, Springer, 719-769.

Witham, C., 2005, Volcanic disasters and incidents: A new database: Journal of Volcanology and Geothermal Research, 148(3), 191-233.

Zanchetta, G., Sulpizio, R., Pareschi, M., Leoni, F., Santacroce, R., 2004, Characteristics of May 5-6, 1998 volcaniclastic debris flows in the Sarno area (Campania, southern Italy): relationships to structural damage and hazard zonation: Journal of volcanology and geothermal research, 133(1), 377-393.

Zúccaro, G., Santo, A., Cacace, F., Gregorio, D., Crescenzo, G., 2012, Building vulnerability assessment under landslides actions: Castellammare di Stabia case study, Italia: Online report of Italian Geological Society, $21,470-472$. 